\title{
Maximum current density and beam brightness achievable by laser-driven electron sources
}

\author{
D. Filippetto, ${ }^{1}$ P. Musumeci, ${ }^{2}$ M. Zolotorev, ${ }^{1}$ and G. Stupakov ${ }^{3}$ \\ ${ }^{1}$ Lawrence Berkeley National Laboratory, One Cyclotron Road, Berkeley, California 94720, USA \\ ${ }^{2}$ Department of Physics and Astronomy, UCLA, Los Angeles, California 90095, USA \\ ${ }^{3}$ SLAC National Accelerator Laboratory, Menlo Park, California 94025, USA
}

(Received 26 April 2013; published 18 February 2014)

\begin{abstract}
This paper discusses the extension to different electron beam aspect ratio of the Child-Langmuir law for the maximum achievable current density in electron guns. Using a simple model, we derive quantitative formulas in good agreement with simulation codes. The new scaling laws for the peak current density of temporally long and transversely narrow initial beam distributions can be used to estimate the maximum beam brightness and suggest new paths for injector optimization.
\end{abstract}

DOI: 10.1103/PhysRevSTAB.17.024201

PACS numbers: 29.25.Bx, 29.20.Ej, 29.27.Bd

Modern photoguns have made a tremendous impact on the range of applications of electron accelerators with the improvement of the source beam brightness by orders of magnitude with respect to previous generations of electron sources. The blending of high peak fields at the cathode with sub-mm spot size and sub-ps short laser pulses have enabled the production of very high current and low emittance beams that can be used to drive fourth generation light sources [1], generate copious amounts of $\mathrm{THz}$ radiation [2], probe structural dynamics at the ultrafast time scale [3], or serve as injectors for laser-based compact accelerators [4]. Despite the extensive use of laser-driven electron guns in a broad range of science applications, a complete theory for the maximum current extractable from such sources has not been derived yet.

The maximum current density in an electron source is typically given by the Child-Langmuir law (C-L, [5]) which is found by self-consistently solving the Poisson equation, the equations of motion for the electrons and the continuity equation in an accelerating gap of voltage $V$. The derivation assumes (i) an infinitely wide beam in the transverse dimensions (1D approximation), (ii) that the beam completely fills the accelerating gap so that a steady state solution can be found, and (iii) that relativistic effects can be neglected.

In state-of-the-art photogun designs, different schemes and field configurations are used in the attempt to maximize the extracted beam brightness with the optimum point strongly depending on the specific requirements of the beam application (for a comprehensive review see [6]). Nevertheless, to take advantage of the time structure of the drive lasers and increase the peak current, in all recently

Published by the American Physical Society under the terms of the Creative Commons Attribution 3.0 License. Further distribution of this work must maintain attribution to the author $(s)$ and the published article's title, journal citation, and DOI. commissioned or designed photoinjector sources the initial electron beam pulse length is always much smaller than the accelerating gap. Furthermore, the laser spot size on the cathode in photoinjectors tends to be small (sub-mm) to decrease the cathode emittance contribution and increase the brightness.

Such conditions are very far from the range of application of the Child-Langmuir formula, with its onedimensional steady state solution. As a matter of fact many photoguns have achieved current densities much higher than those predicted by the C-L [6]. A different formula is required to describe the maximum current density for the cases of experimental interest of sub-ps and ps laser pulses illuminating cathodes with sub-mm spot sizes in the high field region.

Recently a few papers proposed a solution to such a problem in specific areas of interest. For very short bunches, the so-called "pancake regime," the approximation of a deltalike pulse has been adopted [7], where the beam is seen as an infinitely thin sheet of charge, with an associated surface charge density. The problem has also been treated for beam transverse sizes comparable to the accelerating gap dimensions (down to 0.1 times the gap), first by fitting the simulation results [8], and then by solving analytically the electric field integral after a Taylor series expansion [9]. Nevertheless a comprehensive theory valid for all beam aspect ratios and linking together all the different regimes has not been proposed yet.

In this paper we propose a simple physical picture of the problem that leads to a general solution for any beam aspect ratio, and allows us to derive scaling laws for beam current density. Given the connection between the current density and the beam brightness we can then obtain a new scaling law for the maximum brightness achievable in photoinjectors thus expanding the result recently discussed in [7] to beams with different aspect ratios. Such a scaling law gives a quantitative estimate of the relation between the 
various beam parameters. The optimum initial beam shape may indeed not be a temporally short pancakelike distribution. For example, for a fixed charge, a lower extracted current from the cathode allows for a smaller transverse emittance.

It should be noted that if the laser energy on the photocathode is increased to extract peak currents well above the limits given in this paper, a different regime of photocathode physics will occur characterized by current oscillations (a virtual cathode instability) [10]. The direct consequence of such instability is a strong degradation of the beam brightness. The description of this regime is beyond the scope of this paper.

Let us begin our discussion from the classic C-L equation for the limiting current density:

$$
j_{C L, 1 D}=\frac{4 \epsilon_{0}}{9} \sqrt{\frac{2 e}{m}} \frac{V_{0}^{3 / 2}}{d^{2}},
$$

where $V_{0}$ is the total applied voltage in the gap, $d$ is the gap length, $\epsilon_{0}$ is the vacuum dielectric permittivity, $m$ and $e$ are the electron mass and charge.

In order to get a physical picture for the electric field and particle dynamics, we model the beam as formed by infinitely thin disks of surface charge density $\sigma$ which are continuously emitted at the cathode plane. In the C-L picture, the beam longitudinal slices are assumed infinitely wide transversely, thus generating a constant electric field $E_{s}= \pm \sigma / 2 \epsilon_{0}$, only dependent on the sheet charge density but independent of the longitudinal distance from the slice. In this approximation the electric field at the $z=0$ plane (cathode) keeps adding up as more particles are emitted, with the current density eventually reaching the C-L steady state solution where the total electric field (external+ space charge) at the cathode changes sign.

In the case of finite transverse dimensions, the electric field of a thin disk is not constant anymore with the distance from the source. The on-axis field for a disk of radius $R$ is $E_{s}(\zeta)= \pm \frac{\sigma}{2 \epsilon_{0}}\left(1-|\zeta| / \sqrt{\zeta^{2}+R^{2}}\right)[11]$, where $\zeta$ is the onaxis coordinate measured from the center of the disk; it is shown in Fig. 1 together with the field of an infinitely large charged sheet. For a finite $R$, the on-axis electric field dies off quickly for an increasing distance from the disk. This means that particles at a distance larger than $z_{\text {eff }} \sim R$ will not contribute much to direct or the image field at the cathode.

We can then define an effective diode of length $z_{\text {eff }}$, and apply the C-L equation to this new diode geometry, where the gap length $d$ is replaced by $z_{\text {eff }}$. The gap voltage $V_{0}$ for this effective diode can be calculated as $V_{0} \frac{z_{\text {eff }}}{d}=E_{0} z_{\text {eff }}$ which is obtained with the consideration that the potential in the empty part of the gap is unperturbed [12]. $E_{0}$ is the gun accelerating field which is assumed constant in this derivation. This assumption is valid in case of static accelerating fields (DC guns) or laser pulse length much
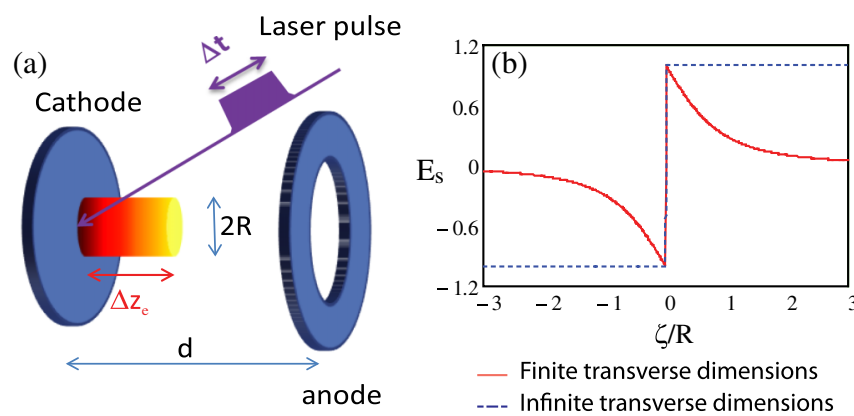

FIG. 1 (color online). (a) Definition of 2D Child-Langmuir parameters. (b) On-axis electric field of a charged disk for infinite (blue dotted) and finite (red dashed) transverse dimensions. The surface charge density is the same in both cases. The on-axis coordinate $\zeta$ from the center of the disk is normalized by the disk radius $R$. The electric field is normalized by $\sigma / 2 \epsilon_{0}$.

smaller than the rf period. The space-charge limiting current for the effective diode can then be expressed using a modified Eq. (1) for the current density and the beam cross section $\pi R^{2}$ as

$$
I_{\text {sat }, 2 D}=C_{c} I_{0} \frac{\sqrt{2}}{9}\left(\frac{e E_{0} R}{m c^{2}}\right)^{\frac{3}{2}}
$$

where $I_{0}=4 \pi \epsilon_{0} m c^{3} / e \simeq 17 \mathrm{kA}$ is the characteristic Alfven current, and $C_{c}$ is a order-of-unity constant that needs to be found by fitting simulation results. A more formal derivation of (2) is given in the Appendix.

Equation (2) shows a dependence of the emitted current on the externally applied electric field instead of the total voltage, which is expected in the approximations of vanishingly small transverse dimensions where the anode can be considered infinitely far away from the cathode.

To verify Eq. (2) we analyze two cases representative of (i) high gradient S-band $\mathrm{rf}$ photoinjectors and (ii) low frequency or DC guns. The electric field and laser pulse length for these two cases are taken to be respectively $E_{0}=60 \mathrm{MV} / \mathrm{m}, \quad \Delta t=10 \mathrm{ps}$ and $E_{0}=20 \mathrm{MV} / \mathrm{m}$, $\Delta t=60 \mathrm{ps}$. Here we consider a transversely uniform laser pulse with flattop longitudinal profile of length $\Delta t$ illuminating the cathode. Although ideal, such laser pulse profiles are not uncommon in laser system designs for driving photoemission in electron sources. Furthermore, in the case of fully space-charge limited emission, the radial intensity profile is flattened out in emission by the saturation. Figure 2 shows the total charge emitted as a function of laser spot radius on the cathode, simulated with the diskmodel simple code (blue triangles), and with threedimensional codes [ASTRA [13] (black squares) and General Particle Tracer [14] (green dots)] using a transverse flattop initial distribution. The red curve shows the theoretical beam charge, obtained as $I_{\mathrm{sat}, 2 D}$ from Eq. (2) times the laser pulse length $\Delta t$. A best fit for the simulated data found a value of $C_{c}=1 \pm 0.1$, implying an effective 

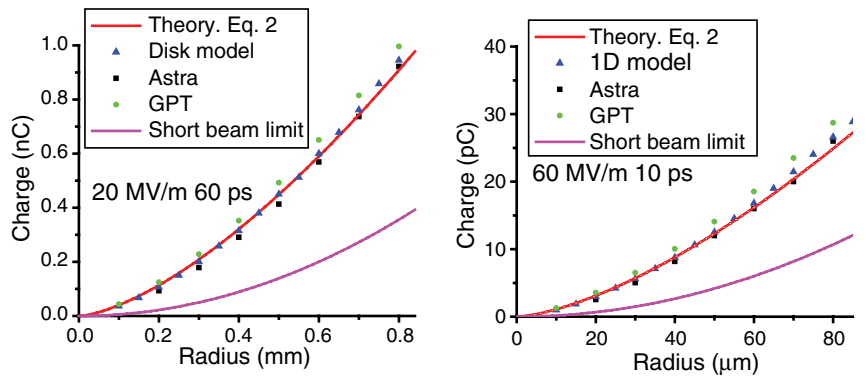

FIG. 2 (color online). Charge extracted as a function of radius. Disk model, three-dimensional simulations (ASTRA and GPT) and theory $\left(C_{c}=1\right)$ for two different cases.

diode extension approximately equal to the beam radius at cathode, i.e., $z_{\text {eff }} \cong R$.

Three-dimensional effects due to the radial dependence of the longitudinal component of the space-charge electric field and to the transverse expansion that the bunch undergoes as it is being emitted have been ignored in the above derivation of Eq. (2). The disk model was used here as an intermediate step to verify the scaling law behavior in an idealized contest. The maximum charge in this model is the integral of all the slices emitted up to the point where the total field at the cathode changes sign. The model only considers the on-axis field of a charged disk, and also does not take into account the transverse dynamics. One could think that a transverse explosion in the cathode vicinity would lower the beam density, playing a determinant role in the extraction. The agreement of the theory and of the disk model with the 3D simulations (see Fig. 2) confirms that these effects are small and do not alter the functional dependence of the emission.

The dependence on the externally applied electric field can also be verified with the simulations, and the results are shown in Fig. 3(a) for a particular case of initial beam radius $R=40 \mu \mathrm{m}$ and $\Delta t=15 \mathrm{ps}$.

Each point of Figs. 2 and 3(a) has been taken simulating a very large laser energy. The amount of extracted charge is found to be independent of the laser energy in the limit
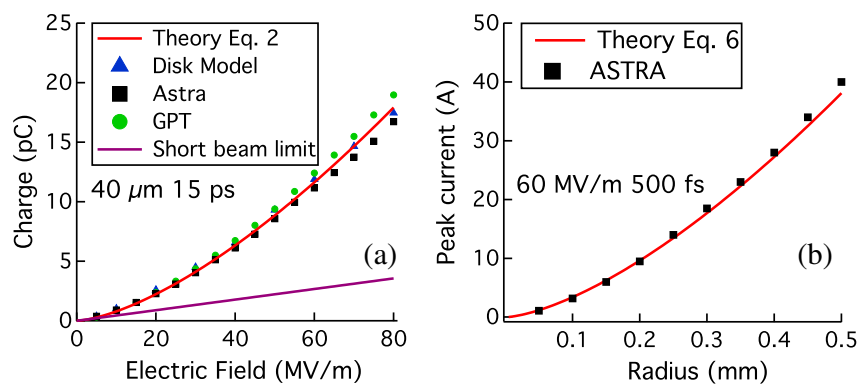

FIG. 3 (color online). (a) Emitted charge vs electric field $E_{0}$ for a cigar aspect ratio beam, $C_{c}=1$. (b) Peak current vs cathode size in the short pancake-beam limit $\left(E_{0}=60 \mathrm{MV} / \mathrm{m}\right.$, $\Delta t=500 \mathrm{fs}$ ) and $C_{s}=1$ [see Eq. (6)].

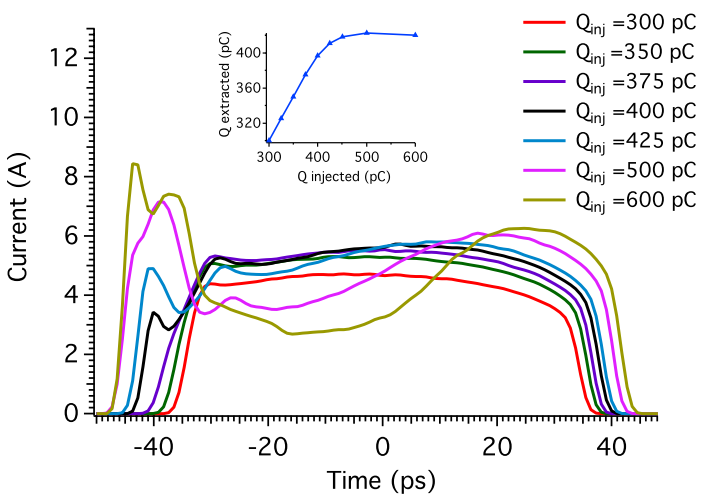

FIG. 4 (color online). Electron beam current extracted as function of charge injected (laser energy) into the diode. The example corresponds to the radius of $500 \mu \mathrm{m}$ in Fig. 2(a). The inset shows the extracted charge vs the injected one. The laser pulse length was fixed to $60 \mathrm{ps}$. The ASTRA code was used in the simulations.

when the emitted charge reaches the space-charge saturation level. This value is compared with the charge predicted by the theory [current value from Eq. (2) multiplied by the laser pulse length $\Delta t$ ]. The evolution of the electron current profile for laser energies above the spacecharge limit is reported in Fig. 4, for a beam corresponding to the point at $R=500 \mu \mathrm{m}$ in Fig. 2(a). The tail of the current profile shows the presence of a second peak and the temporal profiles show the onset of characteristic virtual cathode current oscillations.

Further insight is offered by looking at the total longitudinal electric field and charge density distribution as a function of distance from the cathode for the case of $\Delta t=60 \mathrm{ps}$ and accelerating gradient $E_{0}=20 \mathrm{MV} / \mathrm{m}$ (Fig. 5). The snapshot of the field distribution is taken at a time $60 \mathrm{ps}$ at the tail of the laser pulse i.e., when the last photoelectrons leave the cathode. On the left, we plot a comparison of the fields for a beam of infinitely wide transverse dimensions and one of a finite transverse size $R=400 \mu \mathrm{m}$. The right plot shows the charge density
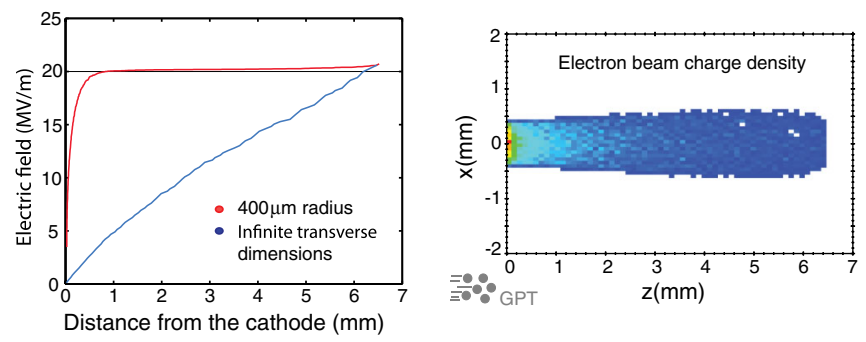

FIG. 5 (color online). Electric field within the electron beam at the end of the laser pulse duration time $\Delta t$ for a finite and infinite transverse dimension (left panel) and the relative electron beam charge density distribution (right panel) for the finite radius case simulated by GPT. The black line on the left panel shows the level of the external field. 
distribution for the finite beam at the same instant, providing an explanation for the behavior of the electric field: all the diode loading occurs over a small distance of the order of $R$. Within this distance the electron beam density rapidly decreases following the C-L theory $\left[\rho(z) \propto z^{-\frac{2}{3}}\right]$, and the total electric field rapidly varies from 0 to the external applied field. Outside this region, the beam dynamics is to a good approximation that of a single particle, and the density only decreases due to the velocityinduced particle redistribution $\left[\rho(z) \propto z^{-\frac{1}{2}}\right]$. The negligible contribution of the beam self-fields to the dynamics outside the effective diode area is confirmed by comparing its amplitude with that of the externally applied field (shown by the black line at $E=E_{0}$ in the left plot). Such a contribution reaches a maximum of $600 \mathrm{kV} / \mathrm{m}$ at the beam's head.

It is important to clarify the role of the image charge in the beam dynamics of space-charge limited emission, by comparing it with a different relevant case. In the blowout regime [15], in order to create a uniformly filled ellipsoidal distribution, the total extracted charge must be kept low enough to minimize the image charge effects. The longitudinal space charge dominates the evolution and the selfelectric field reaches at most few $\mathrm{MV} / \mathrm{m}$ values, and is responsible for a symmetric beam explosion. This regime is very far from what we are considering here, where the image charge is playing a dominant role in the beam evolution, slowing down electrons in the cathode vicinity and affecting the beam density as a function of the distance from the cathode.

Figure 5 provides a better explanation of why the steady state solution gives such a good approximation even though we are looking at an inherently transient phenomenon such as photoemission from a pulsed laser. The electron beam is in fact much longer than the effective length of the diode thus enabling the establishment of a steady state solution within the effective diode.

To be more quantitative, at the end of the laser pulse the beam extends a length $\Delta z_{e}$ into the cathode-anode gap (see Fig. 1) given by

$$
\Delta z_{e}=\frac{e E_{0}}{2 m} \Delta t^{2} .
$$

If $\Delta z_{e}$ is longer than $R$ we are in the "long" bunch (cigar aspect ratio) case, and the approximations leading to Eq. (2) holds. It should be noted that for cases of practical interest and realistic accelerating fields $R$ is typically less than few millimeters, so that for accelerating electric field values less than few hundreds $\mathrm{MV} / \mathrm{m}$ relativistic effects [which will otherwise modify Eq. (3)] can be neglected. The relatively small propagation distance is also the reason why the evolution of the beam transverse dimensions can be neglected and the assumption of constant $R$ is a good approximation.

In the opposite case of short electron bunches (pancake aspect ratio, $\Delta z_{e}<R$ ), a steady state solution cannot be reached since the extraction time is much shorter than the beam effective diode transit time. Nevertheless, a formula for the maximum peak current can still be deduced starting from the maximum surface charge density allowed $\sigma_{\text {sat }}$. The electron beam is approximated as a thin sheet of charge and $\sigma_{\text {sat }}$ can be calculated by imposing the condition that at the end of the laser pulse the electric field at the cathode goes to zero, once again just barely reaching the virtual cathode formation condition where new-born particles would be pushed back into the cathode.

This maximum surface charge density corresponds to a maximum charge,

$$
Q_{\mathrm{sat}}=\sigma_{\mathrm{sat}} \pi R^{2} \cong \epsilon_{0} E_{0} \pi R^{2},
$$

and has been extensively used in analysis of photoinjector performances [7]. It is worth noting the different scaling of the saturated charge with radius and electric field found in the pancake case with respect to the cigar aspect ratio case as shown in Figs. 2(a) and 3(a).

To determine the maximum peak current, we must consider the electron dynamics in the effective diode for an extracted charge density equal to $\sigma_{\text {sat }}$. In this case the total electric field at the cathode will be zero and particles close to the cathode will not move until the density drops. The initial peak current extracted from the cathode will then begin to decrease until the first particles at the head of the bunch exit the diode, thus lowering the electric field at the cathode. The characteristic time for this process to happen,

$$
\tau=\sqrt{\frac{2 m R}{e E_{0}}},
$$

can be used to derive the functional form of the maximum peak current achievable in the pancake regime:

$$
\begin{aligned}
I_{\mathrm{sat}, 2 D}^{\text {short }} & =C_{s} \frac{\sigma_{\mathrm{sat}} \pi R^{2}}{\tau}=C_{s} I_{0} \frac{\sqrt{2}}{9}\left(\frac{e E_{0} R}{m c^{2}}\right)^{3 / 2} \\
& =\frac{C_{s}}{C_{c}} I_{\mathrm{sat}, 2 D},
\end{aligned}
$$

where $C_{s}$ is again a constant to be determined with help of simulations. We note that aside from such order-of-unity constant we re-obtain the same expression of the maximum current density of the long bunch case. Figure 3(b) reports the peak current at the exit of a gun simulated with ASTRA, for the case of $E_{0}=60 \mathrm{MV} / \mathrm{m}$ and $\Delta t=500 \mathrm{fs}$. The red curve represents Eq. (6) for $C_{s}=1$.

Effectively at saturation, the emission time of the last particle in the bunch can be considered equal to $\tau$ [Eq. (5)], even if the real extraction in vacuum happens faster. This characteristic time can be viewed as the cathode-dynamics temporal response to a delta-function laser impulse. 
These formulas clarify the dependence of the final beam properties on the transverse and longitudinal shape of the initial distribution, and have important consequences in the source optimization. The 4D transverse beam brightness is defined as the 4D phase space density of the beam $B_{\perp}=\frac{Q}{\epsilon_{n}^{2}}$, where $\epsilon_{n}$ is the normalized transverse emittance of the beam.

In the limit of cigar aspect ratio we can use Eq. (2) and write a scaling law for $B_{\perp}$ :

$$
B_{\perp} \propto \frac{E_{0}^{3 / 2} \Delta t}{\sqrt{R} \sigma_{p_{\perp}}^{2}}
$$

which reveals that aside from improving the gradient and the excess kinetic energy, significant improvements to the beam quality could be achieved using longer and smaller laser pulses as shown in Fig. 6. In deriving Eq. (7) we have written the normalized transverse emittance for a uniform beam as $\epsilon_{n}=\frac{R}{2} \frac{\sigma_{p_{\perp}}}{m_{0} c}$, and $\frac{\sigma_{p_{\perp}}}{m c}$ is the normalized rms transverse momentum spread of the particles at extraction.

In the pancake limit the total extracted charge is proportional to the square of the beam radius, which makes $B_{\perp}$ independent of the cathode size [7]. Figure 6 plots the $B_{\perp}^{\max }$ as function of cathode size for two different cases. Per definition of long and short bunches adopted, the short and long bunch brightness curves cross for values of the radius given by Eq. (3), for which $\Delta z_{e} \sim R$. To the left of this point we are in the cigar aspect ratio regime and the dashed curve should be used. To the right, we are in the pancake regime and the dotted curve holds.

Even if it is not a fully conserved quantity as properly designed beam lines can effectively manipulate the $6 \mathrm{D}$ phase space beam distribution [16], $B_{\perp}$ is here considered the most appropriate parameter for source characterization. A generalized comparison between electron sources should be done on the basis of the $6 \mathrm{D}$ brightness, taking into account also bunch length and uncorrelated energy spread.

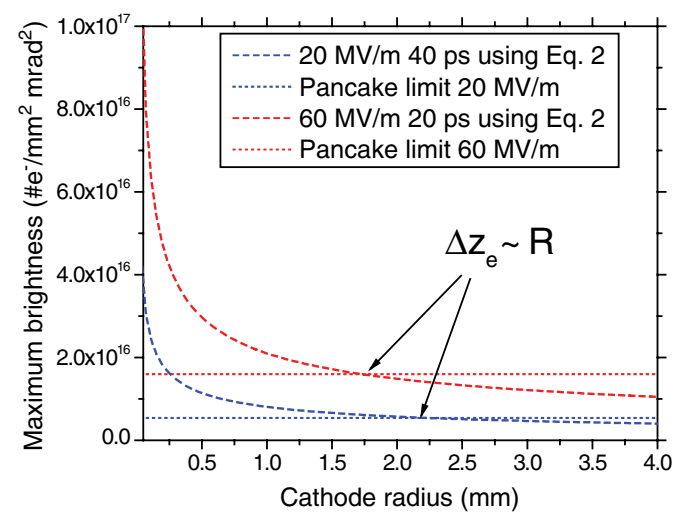

FIG. 6 (color online). Maximum brightness as a function of beam radius. We have assumed a typical value for the normalized rms transverse momentum spread $\frac{\sigma_{p_{\perp}}}{m c}=0.8 \times 10^{-3}$.
Indeed the evolution of the longitudinal emittance is not very well understood in the process of electron beam creation (for example the beam slice energy spread increases by 3 orders of magnitude and its dependence on the beam current needs further studies). Many different mechanisms may play an important role in the evolution of the longitudinal phase space, as rf nonlinearities or intrabeam scattering [17], and the study of the interplay of such effects is beyond the scope of this paper.

Our model provides a description in the region close to the cathode and space-charge effects in the propagation must be taken into account in the evaluation of the beam brightness. It has been shown that in cigar aspect ratio beams some of the space-charge induced emittance growth can be controlled [18]. Nevertheless, as the beam approaches the virtual cathode condition it will become more difficult to preserve the initial brightness [7], therefore an accurate knowledge of the dependence of such condition on beam parameters is essential in the optimization of electron sources.

While Eq. (7) suggests to decrease the beam radius in order to increase the brightness, this is done at expenses of beam current and, therefore, total extracted charge (Fig. 2). If a given output charge $Q_{0}$ is needed, the same equation can be used to obtain a scaling relation between the bunch length and the emittance out of the gun:

$$
\Delta t \propto \frac{1}{\left(E_{0} \epsilon_{n}\right)^{3 / 2}}
$$

In conclusion, a physically transparent expression for the maximum space-charge saturated current density achievable from an electron source has been proposed, valid for beams with dimensions much smaller than the accelerating gap and different aspect ratios, extending the C-L theory to regimes where its assumptions fail. A unified expression, independent of the beam aspect ratio, has been found for the maximum peak current. The different charge scaling for cigar vs pancake beams has been elucidated, leading to different functional forms for the beam brightness. In particular, the maximum transverse brightness for long bunches shows a dependency on the initial pulse length, which could be used as further knob in the source optimization. A smaller transverse emittance for a given charge can be achieved by starting with longer beams. Peak current can be retrieved by longitudinal compression downstream in the accelerator, where the beam is already relativistic. This can be seen as an effective way of trading longitudinal with transverse emittance, shaping the 6D phase space volume.

Our analysis focuses on the dynamics of beam extraction, which takes place within a short region called the "effective diode." The study of such initial dynamics is of great interest as it determines the minimum transverse and longitudinal phase space volumes of the beam. Further 
downstream manipulation of the beam (including beam compression in the gun itself) will only be able to reshape, ideally without increasing, such initial volume. In the hypothesis of constant accelerating field during extraction (wavelength much longer than the pulse length), the treatment is valid for any type of electron source, from DC to rf guns.

The equations reported in this paper give a quantitative tool for the determination of the electron source parameters. This work opens the door to quick and accurate prediction of injector performances in new regimes of operation of electron sources that are of interest to a variety of applications ranging from advanced light sources to time-resolved electron diffraction and microscopy.

The authors would like to thank R. K. Li for stimulating discussions. P. M. acknowledges support from DOE Grants No. DE-FG02-92ER40693, No. DE-FG02-07ER46272, and ONR Grant No. N000140711174. D. F. and M.Z. acknowledge support from DOE Grant No. DE-AC0205CH11231. G.S. acknowledges support from the DOE Grant No. DE-AC02-76SF00515.

\section{APPENDIX: ANALYTICAL DERIVATION OF THE STEADY STATE CURRENT LIMIT IN THE 2D CASE}

As is explained in the main text, for long bunches we can seek a steady state solution, assuming that the current quickly reaches an equilibrium and then does not depend on time. This allows us to use electrostatic potential $\phi$ to represent the electric field inside the bunch in the steady state regime.

Consider a round spot of radius $R$ illuminated by a laser on the cathode. We will use the cylindrical coordinate system $r, \theta, z$ that has the origin at the center of the circle with coordinate $z$ directed to vacuum perpendicular to the surface. Constant electric field $E_{z}=-E_{0}\left(E_{0}>0\right)$ is applied to the cathode. Assume that the illumination is so strong that the emissivity of the surface becomes extremely large, and the current from the surface is limited by the space-charge effects. Denote by $\phi$ the electrostatic potential of the emitted electrons in free space. The total potential $\phi_{\text {tot }}$ is the sum of $\phi$ and the potential of the vacuum electric field $E_{z}, \quad \phi_{\text {tot }}=\phi-E_{z} z=\phi+E_{0} z$. Because of the axisymmetry of the problem $\phi$ does not depend on $\theta, \phi(r, z)$. It satisfies the Poisson equation

$$
\Delta \phi=-\frac{1}{\epsilon_{0}} \rho(r, z),
$$

where $\rho$ is the charge density.

We assume that electrons are moving in $z$ directions only [that is electron's velocity is $\boldsymbol{v}=\left(0,0, v_{z}\right)$ ], then from the charge continuity equation $\rho$ can be expressed through the current density $j_{z}$ on the surface of the cathode, $\rho(r, z)=j_{z}(r) / v_{z}(r, z)$. The velocity $v_{z}$ can be found from the energy conservation (we assume nonrelativistic motion)

$$
v_{z}=\sqrt{\frac{2 e \phi_{\mathrm{tot}}}{m}}=\sqrt{\frac{2 e\left(\phi+E_{0} z\right)}{m}},
$$

where we used the fact that $\phi=0$ at the cathode and neglected the initial energy of the emitted electron. Substituting the expression for $\rho$ into (A1) and using (A2) we obtain

$$
\Delta \phi=-\frac{1}{\epsilon_{0}} j_{z} \sqrt{\frac{m}{2 e\left(\phi+E_{0} z\right)}} .
$$

Since we assume an infinite emissivity of the cathode, this equation should be solved with the boundary condition

$$
\left.\frac{\partial \phi}{\partial z}\right|_{z=0, r \leq R}=-E_{0}
$$

which means that the space-charge electric field of the beam fully compensates for the applied vacuum field $-E_{0}$ on the surface of the cathode. Of course, we also require equipotentiality of the cathode

$$
\phi(r, 0)=0
$$

Let us now introduce dimensionless variables

$$
\begin{aligned}
& \xi=\frac{r}{R}, \quad \zeta=\frac{z}{R}, \quad \psi=-\frac{\phi}{E_{0} R}, \\
& J=-\frac{j_{z}}{\epsilon_{0}} \sqrt{\frac{m R}{2 e}} E_{0}^{-3 / 2},
\end{aligned}
$$

which transforms (A3) in the following one for the function $\psi(\xi, \zeta)$ :

$$
\frac{1}{\xi} \frac{\partial}{\partial \xi} \xi \frac{\partial \psi}{\partial \xi}+\frac{\partial^{2} \psi}{\partial \zeta^{2}}=-J(\xi) \frac{1}{\sqrt{\zeta-\psi}} .
$$

This equation should be solved with the boundary conditions

$$
\left.\frac{\partial \psi}{\partial \zeta}\right|_{\zeta=0, \xi \leq 1}=1, \quad \psi(\xi, 0)=0
$$

The signs of $\psi$ and $J$ in (A6) are chosen in such a way that they are positive.

Equation (A7) with the boundary condition (A8) define a mathematical problem whose solution gives the unknown functions $\psi(\xi, \zeta)$ and $J(\xi)$. Unfortunately, the system (A7) and (A8) cannot be solved analytically, and one has to rely on numerical methods to obtain the solution. The numerical solution of this system is beyond the scope of this paper. 
Note that Eq. (A7) and the boundary conditions (A8) are all dimensionless, hence we expect that the solution of the equation will be such that the function $J$ is of order of 1 , $J \sim 1$. The total current of the beam is obtained by integration of $j_{z}$ over the cross section of the beam,

$$
I=2 \pi \int_{0}^{R} r d r\left|j_{z}\right|=2 \pi \epsilon_{0} \sqrt{\frac{2 e R^{3}}{m}} E_{0}^{3 / 2} \int_{0}^{1} \xi d \xi J .
$$

This result agrees with (2) with $C_{c}=\frac{9}{2} \int_{0}^{1} \xi d \xi J$.

[1] Y. Ding et al. et al., Phys. Rev. Lett. 102, 254801 (2009).

[2] Y. Shen, X. Yang, G. L. Carr, Y. Hidaka, J. B. Murphy, and X. J. Wang, Phys. Rev. Lett. 107, 204801 (2011).

[3] P. Musumeci, J. T. Moody, C. M. Scoby, M. S. Gutierrez, and M. Westfall, Appl. Phys. Lett. 97, 063502 (2010).

[4] X. F. D. Stragier, O. J. Luiten, S. B. van der Geer, M. J. van der Wiel, and G. J. H. Brussaard, J. Appl. Phys. 110, 024910 (2011).

[5] C. D. Child, Phys. Rev. 32, 492 (1911); I. Langmuir, Phys. Rev. 21, 419 (1923).

[6] http://www-bd.fnal.gov/icfabd/Newsletter46.pdf.
[7] I. V. Bazarov, B. M. Dunham, and C. K. Sinclair, Phys. Rev. Lett. 102, 104801 (2009).

[8] J. W. Luginsland, Y. Y. Lau, and R. M. Gilgenbach, Phys. Rev. Lett. 77, 4668 (1996).

[9] Y. Y. Lau, Phys. Rev. Lett. 87, 278301 (2001).

[10] D. H. Dowell, S. Joly, A. Loulergue, J. P. de Brion, and G. Haouat, Phys. Plasmas 4, 3369 (1997).

[11] See D. J. Griffiths, Introduction to Electrodynamics (Prentice-Hall, Englewood Cliffs, NJ, 1999), 3rd ed., Chap. 2.

[12] A. Valfells, D. W. Feldman, M. Virgo, and P. G. O'Shea, Phys. Plasmas 9, 2377 (2002).

[13] K. Flottmann, Astra (DESY, Hamburg, 2000), http://www .desy.de/mpyflo.

[14] The General Particle Tracer, http://www.pulsar.nl/gpt/.

[15] P. Musumeci, J. T. Moody, R. J. England, J. B. Rosenzweig, and T. Tran, Phys. Rev. Lett. 100, 244801 (2008).

[16] M. Cornacchia and P. Emma, Phys. Rev. ST Accel. Beams 5, 084001 (2002).

[17] M. Reiser, Theory and Design of Charged Particle Beams (Wiley-VCH, New York, 2008), 2nd ed., pp. 352, 472-476.

[18] R. K. Li, K. G. Roberts, C. M. Scoby, H. To, and P. Musumeci, Phys. Rev. ST Accel. Beams 15, 090702 (2012). 